\title{
Eficácia da fisioterapia aquática na reabilitação de pacientes idosos com a doença de Parkinson: revisão sistemática
}

\author{
Effectiveness of aquatic physiotherapy on the rehabilitation \\ of elderly patients with Parkinson's disease: a systematic review
}

\author{
Yasmin Varjão Gama Bispo ${ }^{1}$ \\ Adrielle Rizzo dos Santos Vasconcelos Guerra ${ }^{1}$ \\ Dayana da Silva Santos ${ }^{2}$ \\ Igor de Matos Pinheiro ${ }^{3}$
}

\section{RESUMO}

Introdução: Idosos com doença de Parkinson necessitam da reabilitação multiprofissional para minimizar as perdas das habilidades gerais e a incapacidade. A fisioterapia aquática é uma das estratégias utilizadas na reabilitação destes pacientes por ser realizada em um ambiente mais seguro para as atividades, quando comparadas à terapia em solo. Objetivo: Revisar sistematicamente a literatura em relação a eficácia da hidroterapia em pacientes idosos com a doença de Parkinson. Materiais e Métodos: Revisão sistemática realizada nas bases de dados Scielo, Lilacs e Pubmed. Foram incluídos ensaios clínicos publicados no período de janeiro de 2010 a março de 2020, que avaliaram a atuação da fisioterapia aquática em pacientes com doença de Parkinson. Foram excluídos estudos que abordaram a realização da fisioterapia aquática associada a outras intervenções fisioterapêuticas. Avaliou-se a qualidade metodológica dos artigos selecionados por meio da escala PEDro. Resultados: 0 número de participantes nos estudos desta revisão variou entre 9 a 56 idosos, com doença de Parkinson idiopática, nos estágios 1 a 3 da Escala Hoehn e Yahr. A técnica mais utilizada foi a terapia aquática de Ai Chi e a maioria dos estudos realizaram as intervenções da hidroterapia duas vezes por semana. Os principais resultados das intervenções da fisioterapia aquática estão relacionados à melhora do equilíbrio, mobilidade, marcha, diminuição da percepção da dor, além de contribuir na modulação dos níveis BDNF e dos marcadores inflamatórios (MCP-1, IL-1ra e IL-1 ß). Conclusão: A fisioterapia aquática é um método eficaz na reabilitação de idosos nos estágios leve a moderado da doença de Parkinson.

\section{PALAVRAS-CHAVE}

Idoso; Hidroterapia; Doença de Parkinson.

\footnotetext{
${ }^{1}$ Graduanda em Fisioterapia (9o semestre) pela União Metropolitana de Educação e Cultura - UNIME / Lauro de Freitas. ${ }^{2}$ Universidade Federal da Bahia - UFBA.

${ }^{3}$ Fisioterapeuta, Especialista em Reabilitação Neurofuncional, União Metropolitana de Educação e Cultura - UNIME / Lauro de Freitas.
} 


\section{ABSTRACT}

Introduction: Elderly people with Parkinson's disease need multiprofessional rehabilitation to minimize the loss of general skills and disability. Aquatic physitherapy is one of the strategies used in the rehabilitation for those patients because it is performed in a safer environment for the activities, when compared to the therapy on the ground. Objective: To systematically review the literature regarding the effectiveness of hydrotherapy in elderly patients with Parkinson's disease. Materials and Methods: Systematic review performed on Scielo, Lilacs and Pubmed databases. Clinical trials published from January 2010 to March 2020 were included, which evaluated the performance of aquatic physiotherapy in patients with Parkinson's disease. Studies that addressed the performance of aquatic physiotherapy associated with other physiotherapeutic interventions were excluded. The methodological quality of the articles selected was evaluated by the PEDro scale. Results: The number of study participants ranged from 9 to 56 elderly people with idiopathic Parkinson's disease, in stages 1 to 3 of the Hoehn and Yahr Scale. The most used technique was Ai Chi aquatic therapy and most studies performed hydrotherapy interventions twice a week. The main results of Aquatic Physiotherapy interventions are related to improving balance, mobility, gait, decreased pain perception, in addition to contributing to modulation of BDNF levels and inflammatory markers (MCP-1, IL-1ra and IL-1 $\beta$ ). Conclusion: Aquatic physiotherapy is an effective method for the rehabilitation of the elderly from the mild to moderate stages of Parkinson's disease.

\section{KEYWORDS}

Aged; Hydrotherapy; Parkinson Disease. 


\section{INTRODUÇÃo}

A doença de Parkinson (DP) é um distúrbio neurológico progressivo que promove uma perda ou degeneração significativa de células dopaminérgicas dos gânglios da base (HEISTERS, 2011), resultando em uma disfunção da via nigroestriatal, com diminuição da concentração de dopamina ao nível dos receptores dopaminérgicos situados no corpo estriado (MENESES; TEIVE, 1996). No Brasil, a DP atinge em torno de 3\% da população idosa acima de 65 anos (RIEDER et al., 2016).

Os pacientes com DP apresentam sintomas motores que surgem quando já existe perda de $60 \%$ dos neurônios dopaminérgicos. As manifestações mais comuns são o tremor em repouso, rigidez pelo aumento do tônus muscular, bradicinesia e alterações posturais. Os pacientes também apresentam sintomas não motores, como constipação, diminuição do olfato, distúrbios do sono, comprometimento da memória, depressão e distúrbios do sistema nervoso autônomo. Há uma forte associação da depressão e ansiedade nos pacientes com DP (RIEDER et al., 2016, LIMONGI, 2001).

A reabilitação dos pacientes com DP deve ser realizada por uma equipe multiprofissional que visa retardar ou impedir as perdas de habilidades gerais e a incapacidade. o tratamento fisioterapêutico está direcionado para meIhora da mobilidade, da força muscular, do equilíbrio, da aptidão física e da qualidade de vida destes pacientes (KUOPIO et.al., 2000).

A fisioterapia aquática é uma das estratégias utilizadas pelo fisioterapeuta na reabilitação de pacientes com DP (VIVAS J et.al., 2011) devido às propriedades da água como flutuabilidade e viscosidade que promovem um ambiente mais seguro para a atividade física e reduzem o risco de quedas, quando comparadas à terapia em solo. Além disso, o efeito potencial da água aquecida favorece redução da rigidez, um dos sintomas típicos da DP (VIVAS; ARIAS; CUDEIRO, 2011; GEYTENBEEK, 2002).

Diversas técnicas da fisioterapia aquática podem ser utilizadas para a reabilitação de indivíduos com DP, tais como a hidrocinesioterapia, o Ai Chi, Bad Ragaz e Watsu. Com o intuito de avaliar as diferentes técnicas descritas e os seus resultados, o presente estudo tem por objetivo re- visar sistematicamente a literatura em relação à eficácia da hidroterapia em pacientes idosos com a doença de Parkinson.

\section{MATERIAIS E MÉTODOS}

Esta revisão segue as diretrizes para revisões sistemáticas e metanálises PRISMA (GALVÃO; PANSANI; HARRAD, 2015) e aborda a atuação da fisioterapia aquática na reabilitação de pacientes com a doença de Parkinson. Inicialmente foi realizada uma busca nas bases de dados Scielo, Lilacs e Pubmed no mês de março de 2020, utilizando os descritores "Hidroterapia" OR "fisioterapia aquática” AND "doença de Parkinson", e seus correlatos em inglês. Após a busca, dois pesquisadores independentes realizaram a leitura do título e resumo dos materiais para seleção dos artigos conforme os critérios de inclusão e exclusão. Em caso de discordância entre os pesquisadores, um terceiro avaliador foi consultado.

Foram incluídos ensaios clínicos publicados no período de janeiro de 2010 a março de 2020, que avaliaram a atuação da fisioterapia aquática em pacientes com doença de Parkinson. Foram excluídos estudos que abordaram a realização da fisioterapia aquática associada a outras intervenções fisioterapêuticas.

Em cada ensaio clínico, buscou-se identificar as características dos participantes e alocações realizadas, caracterização das intervenções (tipo, frequência, duração) e principais resultados. Para avaliar a qualidade metodológica dos artigos selecionados, foi utilizado a escala PEDro. A escala PEDro é um instrumento adaptado da escala Delphi, composto por onze critérios de avaliação tendo uma pontuação máxima de 10 pontos. (SHIWA et.al., 2011).

\section{RESUTADOS}

Inicialmente foram identificados 37 artigos nas bases de dados pesquisadas, destes, 20 foram excluídos por serem artigos de revisão bibliográfica. Desta maneira, 17 artigos foram selecionados para compor esta revisão sistemática. As características dos ensaios clínicos e a pontuação na Escala PEDro estão apresentadas na Tabela 1. 
Tabela 1. Características dos ensaios clínicos e pontuação na Escala PEDro.

\begin{tabular}{|c|c|c|c|c|}
\hline Autor/ano & $\begin{array}{l}\text { Escala } \\
\text { PEDro }\end{array}$ & $\begin{array}{l}\text { Participantes e grupos } \\
\text { de alocação }\end{array}$ & Intervenção, frequência e duração & $\begin{array}{l}\text { Principais } \\
\text { resultados }\end{array}$ \\
\hline $\begin{array}{l}\text { DE LA CRUZ, } \\
2019\end{array}$ & 7 & $\begin{array}{l}30 \text { indivíduos, } 1 \text { a } 3 \mathrm{HY} \\
-\mathrm{GE}=15,66,80+/- \\
5,267 \text { anos } \\
-\mathrm{GC}=15\end{array}$ & $\begin{array}{l}\text { Exercícios de Aquecimento, Terapia } \\
\text { aquática de Ai Chi, relaxamento } \\
\text { Frequência = } 2 \text { x semana por } 45 \text { minutos } \\
\text { Duração = } 20 \text { sessões / } 10 \text { semanas }\end{array}$ & $\begin{array}{l}\text { Melhora na dor, } \\
\text { equilíbrio e na } \\
\text { capacidade } \\
\text { funcional }\end{array}$ \\
\hline $\begin{array}{l}\text { GERMANOS et } \\
\text { al., } 2019\end{array}$ & 3 & $\begin{array}{l}26 \text { indivíduos } \\
\text { - GE = } 12 \text { com DP } \\
\text { idiopática, } 65,08 \pm 6,73 \\
\text { anos } \\
\text { - GC = } 14 \text { indivíduos } \\
\text { saudáveis }\end{array}$ & $\begin{array}{l}\text { Treino aeróbico e de resistência com } \\
\text { motivação verbal: aquecimento, } \\
\text { exercícios resistidos, exercícios de dupla } \\
\text { tarefa, relaxamento. } \\
\text { Frequência = } 2 \text { x semana por } 60 \\
\text { Duração = } 1 \text { mês }\end{array}$ & $\begin{array}{l}\text { Redução dos } \\
\text { níveis de BDNF } \\
\text { em } 48 \text { horas após } \\
\text { a sessão, porém } \\
\text { sem efeitos a } \\
\text { longo prazo }\end{array}$ \\
\hline $\begin{array}{l}\text { SILVA; ISRAEL, } \\
2019\end{array}$ & 5 & $\begin{array}{l}25 \text { indivíduos com DP } \\
\text { idiopática, } 1 \text { a } 4 \text { HY } \\
\text { - GE = 14, 63,12 } \pm 13,61 \\
\text { anos } \\
\text { - GC = } 11\end{array}$ & $\begin{array}{l}\text { Exercícios aquáticos de dupla tarefa } \\
\text { Frequência = } 2 \text { x semana por } 60 \text { minutos } \\
\text { Duração = } 20 \text { sessões } / 10 \text { semanas }\end{array}$ & $\begin{array}{l}\text { Melhora } \\
\text { mobilidade } \\
\text { funcional, } \\
\text { equilíbrio e } \\
\text { marcha }\end{array}$ \\
\hline $\begin{array}{l}\text { DE LA CRUZ, } \\
2018\end{array}$ & 6 & $\begin{array}{l}29 \text { indivíduos, } 1 \text { a } 3 \text { HY } \\
- \text { GE }=14,65,87 \pm 7,09 \\
\text { anos } \\
\text { - GC = } 15\end{array}$ & $\begin{array}{l}\text { Aquecimento, Terapia aquática em } \\
\text { grupo de Ai Chi e Resfriamento } \\
\text { Frequência = } 2 \text { x semana por } 45 \\
\text { Duração = } 22 \text { sessões / } 11 \text { semanas }\end{array}$ & $\begin{array}{l}\text { Redução da dor, } \\
\text { bradicinesia e } \\
\text { rigidez articular e } \\
\text { melhor da } \\
\text { qualidade de vida }\end{array}$ \\
\hline $\begin{array}{l}\text { POCHMANN et } \\
\text { al., } 2018\end{array}$ & 6 & $\begin{array}{l}26 \text { indivíduos } \\
\text { - GE = } 12 \text { pacientes com } \\
\text { DP idiopática, } 65,08 \pm \\
6,73 \text { anos } \\
\text { - GC = } 14 \text { indivíduos } \\
\text { saudáveis }\end{array}$ & $\begin{array}{l}\text { Exercícios aquáticos aeróbicos em } \\
\text { grupo, resistidos, com integração e } \\
\text { relaxamento } \\
\text { Frequência = } 2 \text { x semana por } 60 \text { minutos } \\
\text { Duração = } 1 \text { mês }\end{array}$ & $\begin{array}{l}\text { Atenuação das } \\
\text { respostas } \\
\text { imunológicas em } \\
\text { indivíduos com } \\
\text { DP em uma } \\
\text { perspectiva de } \\
\text { curto e longo } \\
\text { prazo }\end{array}$ \\
\hline $\begin{array}{l}\text { CARROLL et al., } \\
2017\end{array}$ & 6 & $\begin{array}{l}21 \text { indivíduos com DP } \\
\text { idiopática, } 1 \text { a } 3 \mathrm{HY} \\
\text { - GE }=11,71,42 \pm 4,9 \\
\text { anos } \\
\text { - GC = } 10 \text { cuidados } \\
\text { usuais }\end{array}$ & $\begin{array}{l}\text { Aquecimento cardiovascular e } \\
\text { alongamento, treino de marcha, } \\
\text { resfriamento } \\
\text { Frequência = } 2 \text { x semana por } 45 \text { minutos } \\
\text { Duração = } 12 \text { sessões / } 6 \text { semanas }\end{array}$ & $\begin{array}{l}\text { Melhora na } \\
\text { deficiência } \\
\text { motora. Não } \\
\text { foram observadas } \\
\text { diferenças } \\
\text { significativas } \\
\text { entre os grupos } \\
\text { para alterações } \\
\text { na marcha }\end{array}$ \\
\hline $\begin{array}{l}\text { DE LA CRUZ et } \\
\text { al., } 2017\end{array}$ & 3 & $\begin{array}{l}15 \text { indivíduos com DP, } 1 \\
\text { a } 3 \mathrm{HY} \\
\text { - } \mathrm{GE}=15,66,80 \pm 5.267 \\
\text { anos } \\
\text { - } \mathrm{GC}=15\end{array}$ & $\begin{array}{l}\text { Terapia aquática de Ai Chi } \\
\text { Frequência = } 2 \text { x semana por } 30 \text { a } 45 \\
\text { minutos } \\
\text { Duração = } 10 \text { semanas }\end{array}$ & $\begin{array}{l}\text { Melhora } \\
\text { significativa nos } \\
\text { escores para } \\
\text { percepção da dor, } \\
\text { equilíbrio e } \\
\text { marcha com } \\
\text { benefícios após } \\
\text { um mês }\end{array}$ \\
\hline
\end{tabular}




\begin{tabular}{|c|c|c|c|c|}
\hline Autor/ano & $\begin{array}{l}\text { Escala } \\
\text { PEDro }\end{array}$ & $\begin{array}{l}\text { Participantes e grupos } \\
\text { de alocação }\end{array}$ & Intervenção, frequência e duração & $\begin{array}{l}\text { Principais } \\
\text { resultados }\end{array}$ \\
\hline $\begin{array}{l}\text { KURT et al., } \\
2017\end{array}$ & 8 & $\begin{array}{l}40 \text { indivíduos com DP } \\
\text { idiopática, } 2 \text { a } 3 \text { HY } \\
\text { - GE }=20,62,41 \pm 6,76 \\
\text { anos } \\
- \text { GC }=20\end{array}$ & $\begin{array}{l}\text { Exercícios de Aquecimento, Terapia } \\
\text { aquática de Ai Chi, relaxamento } \\
\text { Frequência }=5 \text { x semana por } 60 \text { minutos } \\
\text { Duração = } 25 \text { sessões / } 5 \text { semanas }\end{array}$ & $\begin{array}{l}\text { Melhora do } \\
\text { equilíbrio, da } \\
\text { mobilidade, da } \\
\text { habilidade } \\
\text { motora e }\end{array}$ \\
\hline $\begin{array}{l}\text { VOLPE et al., } \\
2017\end{array}$ & 6 & $\begin{array}{l}30 \text { indivíduos com } \mathrm{DP},< \\
3 \mathrm{HY} \\
-\mathrm{GE}=15,76,7 \pm 4,0 \\
\text { anos } \\
-\mathrm{GC}=15\end{array}$ & $\begin{array}{l}\text { Exercícios aquáticos } \\
\text { Frequência = } 5 \text { x semana por } 60 \text { minutos } \\
\text { Duração = } 8 \text { semanas }\end{array}$ & $\begin{array}{l}\text { qualidade de vida } \\
\text { Melhora } \\
\text { significativa dos } \\
\text { parâmetros } \\
\text { têmpora } \\
\text { espaciais da } \\
\text { marcha, da } \\
\text { postura do tronco } \\
\text { no plano sagital e } \\
\text { coronal }\end{array}$ \\
\hline $\begin{array}{l}\text { VOLPE et al., } \\
2017\end{array}$ & 6 & $\begin{array}{l}56 \text { indivíduos com DP, } 1 \\
\text { a } 3 \text { HY } \\
\text { Coorte } 1 \\
\text { - GE }=24 \\
\text { - GC }=12 \\
\text { Coorte } 2 \\
\text { - } \mathrm{GE}=10 \\
\text { - GC }=10\end{array}$ & $\begin{array}{l}\text { Protocolo 1: caminhada com a água na } \\
\text { linha mamilar } \\
\text { Protocolo 2: caminhada na água em } \\
\text { velocidade selecionada } \\
\text { Frequência = } 7 \text { x semana } \\
\text { Duração = } 21 \text { dias }\end{array}$ & $\begin{array}{l}\text { Melhora } \\
\text { significativa da } \\
\text { velocidade da } \\
\text { marcha nos dois } \\
\text { protocolos }\end{array}$ \\
\hline $\begin{array}{l}\text { ZHU et al., } \\
2017\end{array}$ & 7 & $\begin{array}{l}46 \text { indivíduos com DP, } 2 \\
\text { a } 3 \mathrm{HY} \\
\text { - } \mathrm{GE}=23,65 \pm 6 \text { anos } \\
\text { - } \mathrm{GC}=23\end{array}$ & $\begin{array}{l}\text { Aquecimento, terapia aquática de } \\
\text { obstáculos, resfriamento } \\
\text { Frequência }=5 \text { x semana } \\
\text { Duração = } 30 \text { sessões / } 6 \text { semanas }\end{array}$ & $\begin{array}{l}\text { A terapia } \\
\text { aquática com } \\
\text { obstáculos foi } \\
\text { mais eficaz do } \\
\text { que os protocolos } \\
\text { tradicionais de } \\
\text { marcha e } \\
\text { equilíbrio e o } \\
\text { efeito dura seis } \\
\text { meses }\end{array}$ \\
\hline $\begin{array}{l}\text { VOLPE et al., } \\
2014\end{array}$ & 6 & $\begin{array}{l}34 \text { indivíduos com DP } \\
\text { idiopática, } 2,5 \text { a } 3 \mathrm{HY} \\
-\mathrm{GE}=17,68 \pm 7 \text { anos } \\
-\mathrm{GC}=17\end{array}$ & $\begin{array}{l}\text { Aquecimento cardiovascular, } \\
\text { alongamentos, treino de equilíbrio e } \\
\text { relaxamento } \\
\text { Frequência = } 5 \text { x semana por } 60 \text { minutos } \\
\text { Duração = } 2 \text { meses }\end{array}$ & $\begin{array}{l}\text { Melhora na } \\
\text { estabilidade } \\
\text { postural } \\
\text { reduzindo as } \\
\text { taxas de quedas }\end{array}$ \\
\hline $\begin{array}{l}\text { RODRIGUEZ et } \\
\text { al., } 2013\end{array}$ & 3 & $\begin{array}{l}9 \text { indivíduos, com DP } \\
\text { idiopática, } 1 \text { a } 3 \mathrm{HY} \\
\text { - } \mathrm{GE}=9,67,44 \pm 6,72 \\
\text { anos }\end{array}$ & $\begin{array}{l}\text { Aquecimento, exercícios para equilíbrio, } \\
\text { coordenação e força de membros } \\
\text { inferiores, flutuação passiva } \\
\text { alongamento } \\
\text { Frequência = } 1 \text { x semana } \\
\text { Duração = } 5 \text { meses }\end{array}$ & $\begin{array}{l}\text { Aumento } \\
\text { significativo na } \\
\text { velocidade de } \\
\text { caminhada e no } \\
\text { comprimento da } \\
\text { passada, bem } \\
\text { como na razão de } \\
\text { apoio unipodal / } \\
\text { bipodal }\end{array}$ \\
\hline $\begin{array}{l}\text { SILVA et al., } \\
2013\end{array}$ & 3 & $\begin{array}{l}13 \text { pacientes com } \mathrm{DP} \\
\text { idiopática, } 1 \text { a } 3 \mathrm{HY} \\
\text { - } \mathrm{GE}=13,60 \pm 10 \text { anos }\end{array}$ & $\begin{array}{l}\text { Aquecimento, alongamento, exercícios } \\
\text { ativos e proprioceptivos, relaxamento/ } \\
\text { socialização } \\
\text { Frequência = } 2 \text { x semana por } 60 \text { minutos } \\
\text { Duração = } 16 \text { sessões }\end{array}$ & $\begin{array}{l}\text { Melhora na } \\
\text { qualidade de vida }\end{array}$ \\
\hline
\end{tabular}




\begin{tabular}{|c|c|c|c|c|}
\hline Autor/ano & $\begin{array}{l}\text { Escala } \\
\text { PEDro }\end{array}$ & $\begin{array}{l}\text { Participantes e grupos } \\
\text { de alocação }\end{array}$ & Intervenção, frequência e duração & $\begin{array}{l}\text { Principais } \\
\text { resultados }\end{array}$ \\
\hline $\begin{array}{l}\text { AYÁN; } \\
\text { CANCELA, } \\
2012\end{array}$ & 7 & $\begin{array}{l}20 \text { indivíduos com DP } \\
\text { idiopática, } 1 \text { a } 3 \mathrm{HY} \\
\text { - GE1 = } 1068,9 \pm 9,6 \\
\text { anos } \\
\text { - GE2 = } 1071,9 \pm 5,1 \\
\text { anos }\end{array}$ & $\begin{array}{l}\text { Exercícios aquáticos de baixa } \\
\text { intensidade (grupo } 1 \text { ) } \\
\text { Exercícios aquáticos de resistência } \\
\text { muscular (grupo } 2 \text { ) } \\
\text { Frequência = } 2 \text { x semana por } 60 \text { minutos } \\
\text { Duração = } 12 \text { semanas }\end{array}$ & $\begin{array}{l}\text { Ambas as } \\
\text { intervenções } \\
\text { podem ter efeitos } \\
\text { benéficos na } \\
\text { qualidade de } \\
\text { vida, contudo os } \\
\text { exercícios } \\
\text { aquáticos de } \\
\text { resistência } \\
\text { muscular levaram } \\
\text { a maiores } \\
\text { mudanças na } \\
\text { mobilidade } \\
\text { funcional e } \\
\text { sintomas motores }\end{array}$ \\
\hline $\begin{array}{l}\text { VIVAS; ARIAS; } \\
\text { CUDEIRO, } \\
2011\end{array}$ & 6 & $\begin{array}{l}11 \text { indivíduos com DP } \\
\text { idiopática, } 1 \text { a } 3 \mathrm{HY} \\
\text { - GE1 = } 1068,9 \pm 9,6 \\
\text { anos } \\
\text { - GE2 = } 1071,9 \pm 5,1 \\
\text { anos }\end{array}$ & $\begin{array}{l}\text { Aquecimento, exercícios de mobilidade } \\
\text { de tronco, treino de estabilidade } \\
\text { postural, mudanças de posições } \\
\text { corporais } \\
\text { Frequência = } 2 \text { x semana por } 45 \text { minutos } \\
\text { Duração = } 4 \text { semanas }\end{array}$ & $\begin{array}{l}\text { Melhora } \\
\text { significativa na } \\
\text { estabilidade } \\
\text { postural, nas } \\
\text { atividades de } \\
\text { alcance funcional } \\
\text { e equilíbrio. }\end{array}$ \\
\hline
\end{tabular}

0 número de participantes nos estudos variou entre 9 a 56 indivíduos com doença de Parkinson idiopática nos estágios de 1 a 3 da Escala Hoehn e Yahr. Dentre as principais técnicas da fisioterapia aquática utilizadas, a terapia aquática de Ai Chi foi objeto de estudo em 04 artigos sendo que, antes da aplicação da técnica, foram realizados exercícios de aquecimento e as sessões foram finalizadas com técnicas de relaxamento. Três artigos foram inespecíficos quanto ao tipo de exercícios aquáticos realizados. Em relação à frequência das intervenções, 11 ensaios clínicos realizaram as intervenções duas vezes por semana e três na frequência de cinco vezes por semana. Cada sessão variou de 45 a 60 minutos. Os principais resultados das intervenções da fisioterapia aquática estão relacionados à melhora do equilíbrio, mobilidade funcional e da marcha - 8 artigos; melhora dos domínios da qualidade de vida - 4 artigos. Dois estudos apresentaram redução do BDNF e de citocinas pro-inflamatórias (MCP-1 e IL-1 $\beta$ ) a curto prazo. Em relação à qualidade metodológica dos estudos, 12 artigos apresentaram pontuação $\geq 6$ na Escala PEDro.

\section{DISCUSSÃO}

As intervenções da fisioterapia aquática, apresentadas nos artigos que compõem esta revisão sistemática, promovem melhora nos sintomas da DP, tendo maior relevância naqueles relacionados com a qualidade motora, promovendo uma melhor qualidade de vida nestes idosos. A eficácia do treinamento aquático foi observada nos idosos com DP idiopática nos estágios de leve a moderado da Escala Hoehn e Yahr (SCHENKMAN et al., 2001), e seus benefícios se mantém por até um mês pós intervenção (DE LA CRUZ, 2017).

A terapia aquática com o Ai Chi foi o método mais utilizado nos ensaios clínicos, por ser uma técnica desenvolvida com objetivo de melhorar o controle do equilíbrio, coordenação e estabilização do tronco, além de promover alívio da dor (KONNO, 1997). Em comparação com a terapia em solo, o Ai Chi demonstrou uma evolução mais pronunciada nos pacientes com DP (KURT et al., 2018), além de resultar em uma redução nos valores da Escala UPDRS. Estes resultados positivos na reabilitação foram mantidos após um mês de conclusão do tratamento (DE 
LA CRUZ, 2017). O Ai Chi também reduz significativamente os sintomas motores, bradicinesia e rigidez nos pacientes parkinsonianos (DE LA CRUZ, 2018)

0 ganho no controle do equilíbrio demonstrou ser maior com os exercícios realizados no ambiente aquático em comparação ao ambiente terrestre, pois o ambiente aquático permite o treino do equilíbrio em condições seguras, por diminuir o risco de quedas (VOLPE et al., 2014). As propriedades físicas da água geram uma maior estabilidade na postura, favorecendo a aplicabilidade de técnicas que promovem uma melhor mobilidade. As forças resistivas do ambiente aquático como a viscosidade e turbulência também contribuem para um ganho de mobilidade funcional (AYÁN; CANCELA, 2012).

A fisioterapia aquática demonstrou ser benéfica na melhora da marcha com o aumento no comprimento da passada, na velocidade e no maior tempo de apoio unipodal (RODRÍGUEZ et al, 2013). Os exercícios aquáticos com obstáculos realizado em piscina terapêutica promoveram diminuição dos episódios do freezing, redução da cadência e aumento no comprimento da passada, aumentando assim a velocidade da caminhada. Estas condições contribuem para uma marcha mais segura e com maior controle postura. Salienta-se que as melhorias apresentadas puderam ser observadas em até 6 meses após a execução das atividades (ZHU et al., 2018; RODRÍGUEZ et.al, 2013).

Em relação aos sintomas não motores da DP, a fisioterapia aquática demonstrou eficácia na melhora do humor. Sabe-se que o humor tem impacto negativo na qualidade de vida dos pacientes com doença de Parkinson, com maior chance de desenvolver quadros depressivos, comprometendo a reabilitação (CLERICI et.al., 2019; DE LA CRUZ, 2019). A melhora do humor está associada ao treinamento aquático em um ambiente em que o paciente pode realizar atividades com mais independência frente a propriedade física do empuxo, e também pela realização destas atividades em grupo favorecendo a socialização.

Os ensaios clínicos desta revisão sinalizam uma melhora na qualidade de vida dos participantes independente do programa realizado. A melhora dos domínios físico e da mobilidade podem influenciar nos aspectos psicológicos e emocionais relacionados à DP. A terapia aquática por meio de um ambiente lúdico e de interação social, também pode ter sido um fator importante para estes resultados (ZHU et.al., 2018).
Alterações a curto prazo nos níveis plasmáticos de fator neurotrófico derivado do cérebro (BDNF) foram observadas após a realização de um protocolo de fisioterapia aquática. Após oito semanas de treinamento intermediário, foi possível observar uma melhora na rigidez e diminuição do tônus muscular, contudo, estes resultados não foram mantidos a longo prazo, demonstrando que a modulação dos níveis de BDNF são dependentes da duração do protocolo fisioterapêutico realizado (GERMANOS et al., 2019).

Ao observar os níveis plasmáticos de marcadores inflamatórios (MCP-1, IL-1ra e IL-1 $\beta$ ), foi possível concluir que os pacientes com DP apresentaram níveis aumentados de MCP-1 e níveis reduzidos de IL-1ra, sugerindo que essas citocinas podem ser importantes biomarcadores na DP. Os níveis de MCP-1 e IL-1 $\beta$ sofreram uma redução significativa após o exercício aquático. No que se refere a citocina IL-1ra, foi possível observar que a modulação deste marcador inflamatório imediatamente após a primeira sessão de exercício depende estritamente de aspectos como protocolo, população e compartimento de fluidos avaliados. Desta maneira, a fisioterapia aquática demonstra ser uma intervenção potencial capaz de atenuar as respostas imunes em indivíduos com DP (POCHMANN et al., 2018).

A maioria dos estudos atingiu uma pontuação satisfatória na qualidade metodológica avaliada através da escala PEDro. Doze artigos apresentaram uma pontuação $\geq 6$ e quatro artigos uma pontuação $\leq 5$. Assim, é possível afirmar que grande parte dos ensaios clínicos selecionados para a realização desta revisão sistemática possui uma boa qualidade metodológica, trazendo uma maior confiabilidade nos resultados apresentados.

Não foram encontrados estudos com pacientes com DP em fase avançada da doença não sendo possível conhecer a eficácia da fisioterapia aquática nestes indivíduos. A ausência destes estudos impossibilita uma visão ampla da efetividade da hidroterapia em todos os estágios da DP. Ademais, apenas um estudo evidenciou os efeitos da fisioterapia aquática sobre os níveis de BDNF em pacientes com DP, e um outro estudo evidenciou a modulação de citocinas em resposta a terapia aquática. Recomenda-se a realização de novas pesquisas que considerem estas variáveis e possuam qualidade metodológica adequada. 


\section{CONCLUSÃO}

Esta revisão sistemática demonstrou que a fisioterapia aquática possui eficácia na redução dos sintomas motores que envolvem o equilíbrio, mobilidade, marcha, diminuição da percepção da dor, além de contribuir na modulação dos níveis BDNF e dos marcadores inflamatórios (MCP-1, IL-1ra e IL-1 $\beta$ ) em idosos nos estágios leve a moderado da doença de Parkinson. A terapia aquática também contribuiu na melhora do humor e da qualidade de vida, seja pela realização das técnicas na piscina terapêutica quanto pela realização destas atividades em grupo. 


\section{REFERÊNCIAS}

AYÁN, C.; CANCELA, J. Feasibility of 2 different water-based exercise training programs in patients with Parkinson's disease: a pilot study. Arch Phys Med Rehabil., v. 93, n. 10, p. 1709$1714,2012$.

CARROLL, L. M.; VOLPE, D.; MORRIS, M. E.; SAUNDERS, J.; CLIFFORD, A. M. Aquatic exercise therapy for people with Parkinson disease: a randomized controlled trial. Arch Phys Med Rehabil., v. 98, n. 4, p. 631-638, 2017.

CLERICI, I. et al. Land plus aquatic therapy versus land-based rehabilitation alone for the treatment of freezing of gait in Parkinson disease: a randomized controlled trial. Phys Ther., v. 99, n. 5, p. 591-600, 2019.

DE LA CRUZ, S. P. Effectiveness of aquatic therapy for the control of pain and increased functionality in people with Parkinson's disease: a randomized clinical trial. Eur J Phys Rehabil Med., v. 53, n. 6, p. 825-832, 2017.

DE LA CRUZ, S. P. A bicentric controlled study on the effects of aquatic Ai Chi in Parkinson disease. Complement Ther Med. v. 36, p. 147-153, 2018.

DE LA CRUZ, S. P. Mental health in Parkinson's disease after receiving aquatic therapy: A clinical trial. Acta Neurol Belg., v. 119, n. 2, p. 193-200, 2019.

GALVÃO, T. F.; PANSANI, T. S. A.; HARRAD, D. Principais itens para relatar Revisões sistemáticas e Meta-análises: A recomendação PRISMA. Epidemiol Serv Saúde, v. 24, p. 335-342, 2015.

GERMANOS, S. S. et al. The impact of an aquatic exercise program on BDNF levels in Parkinson's disease patients: short-and long-term outcomes. Funct Neurol., v. 34, n. 2, p. 65-70, 2019.

GEYTENBEEK, J. Evidence for effective hydrotherapy. Physiotherapy, v. 88, n. 9, p. 514-529, 2002.

HEISTERS, D. Parkinson's: symptoms, treatments and research. Br J Nurs., v. 20, n. 9, p. 548-554, 2011.

KONNO, J. Ai chi: a symphony for my body. Physical Therapy Products, v. 2, p. 46-8, 1997.

KUOPIO, A. M.; MARTTILA, R. J.; HELENIUS, H.; TOIVONEN, M.; RINNE, U. K. The quality of life in Parkinson's disease. Mov Disord., v. 15, n. 2, p. 216-223, 2000.

KURT, E. E.; BÜYÜKTURAN, B.; BÜYÜKTURAN, O.; ERDEM, H. R.; TUNCAY, F. Effects of Ai Chi on balance, quality of life, functional mobility, and motor impairment in patients with Parkinson's disease. Disabil Rehabil., v. 40, n. 7, p. 791-797, 2018.

LIMONGI, J. C. P. Conhecendo melhor a doenca de parkinson: uma abordagem multidisciplinar com orientações práticas para o dia-a-dia. São Paulo: Plexus, 2001.

MENESES, M. S.; TEIVE, H. A. G. Histórico. In: Meneses M. S, Teive, H. A.G. Doença de Parkinson: aspectos clínicos e cirúrgicos. Rio de Janeiro: Guanabara Koogan, 1996. p. 4-14.
POCHMANN, D. et al. Cytokine modulation in response to acute and chronic aquatic therapy intervention in Parkinson disease individuals: A pilot study. Neurosci Lett., v. 674, p. 30-35, 2018.

RIEDER, C. R. M. et al. Entendendo a doença de Parkinson: Informações para pacientes, familiares e cuidadores. Porto Alegre: EDIPUCRS, 2016, 179 p.

RODRÍGUEZ, P.; CANCELA, J. M.; AYÁN, C.; DO NASCIMENTO, C.; SEIJO-MARTÍNEZ, M. Efecto del ejercicio acuático sobre la cinemática del patrón de marcha en pacientes con enfermedad de Parkinson: un estudio piloto. Rev Neurol., v. 56, n. 315, p. 20, 2013.

SCHENKMAN, M. L.; CLARK, K.; XIE, T.; KUCHIBHATLA, M.; SHINBERG, M.; RAY, L. Spinal movement and performance of a standing reach task in participants with and without Parkinson disease. Phys Ther., v. 81, n. 8, p. 1400-1411, 2001.

SHIWA, S. R.; COSTA, L. O. P.; MOSER, A. D. L.; AGUIAR, I. C.; OLIVEIRA, L. V. F. PEDro: a base de dados de evidências em fisioterapia. Fisioter Mov., v. 24, n. 3, p. 523-533, 2011.

SILVA, D. M. et al. Efeitos da fisioterapia aquática na qualidade de vida de sujeitos com doença de Parkinson. Fisioter Pesqui., v. 20, n. 1, p. 17-23, 2013.

SILVA, A. Z.; ISRAEL, V. L. Effects of dual-task aquatic exercises on functional mobility, balance and gait of individuals with Parkinson's disease: a randomized clinical trial with a 3-month follow-up. Complement Ther Med., v. 42, p. 119-124, 2019.

VIVAS, J.; ARIAS, P.; CUDEIRO, J. Aquatic therapy versus conventional land-based therapy for Parkinson's disease: an open-label pilot study. Arch Phys Med Rehabil., v. 92, n. 8, p. 1202 1210, 2011.

VOLPE, D.; GIANTIN, M. G.; MAESTRI, R.; FRAZZITTA G. Comparing the effects of hydrotherapy and land-based therapy on balance in patients with Parkinson's disease: a randomized controlled pilot study. Clin Rehabil., v. 28, n. 12, p. 1210-1217, 2014.

VOLPE, D. et al. Underwater gait analysis in Parkinson's disease. Gait Posture, v. 52, p. 87-94, 2017.

VOLPE, D. et al. Water-based vs. non-water-based physiotherapy for rehabilitation of postural deformities in Parkinson's disease: a randomized controlled pilot study. Clin Rehabil.,v. 31, n. 8, p. 1107-1115, 2017.

ZHU, Z. et al. Aquatic obstacle training improves freezing of gait in Parkinson's disease patients: a randomized controlled trial. Clin Rehabil., v. 32, n. 1, p. 29-36, 2018.

FINANCIAMENTO: FUNADESP - Fundação Nacional de Desenvolvimento do Ensino Superior Particular 